\title{
Synthesis, characterisation and antioxidant properties of ferulate-based protic ionic liquids: experimental and modelling approaches
}

\begin{abstract}
Active pharmaceutical ingredients (APIs) in the form of crystalline solid are commonly produced in drug manufacturing industries. However, crystalline solid APIs have a low solubility and the ability to cause polymorphism transformation. In order to overcome these problems, a modification of APIs in the form of protic ionic liquids (PILs) has been proposed. In the present study, a series of five ferulate-based PILs were successfully synthesised and characterised by appropriate spectral analysis. The antioxidant activity of synthesised PILs was determined through the 2,2-diphenyl-1-picrylhydrazyl (DPPH) free radical scavenging assays. The spectroscopy analysis confirmed the formation of PILs supported by the Density Function Theory (DFT) calculation. The synthesised ferulate-based PILs exhibited low $\mathrm{EC}_{50}$ ranging from $12.93 \pm 0.05$ to $17.40 \pm 0.04 \mu \mathrm{M}$ indicating a strong antioxidant activity compared to parent acid $\left(\mathrm{EC}_{50}\right.$ of $\left.21.40 \pm 0.05 \mu \mathrm{M}\right)$. Prediction of antioxidant activity of the PILs and their ionic interactions at the molecular level were studied by applying DFT calculation at B3-LYP/6-311G*** level and COSMO-RS. Based on the optimised geometry, the ion-pair of PILs formed a strong directional intermolecular hydrogen bond between the $-\mathrm{NH}$ group of the cation with the $-\mathrm{COOH}$ group of the anion with interaction energy of -111 to $-120 \mathrm{kcal}$ mol-1. Predicted antioxidant activity indicated that the PILs's cation has a weak interaction with an anion as observed from the $\sigma$-potential analysis.
\end{abstract}

Keyword: DFT calculation; COSMO-RS;Protic ionic liquids; Antioxidant; DPPH radical scavenging 